\title{
Scalability in Vertical Handoff for Wi-Fi and Bluetooth Networks
}

\author{
G G Chitkote \\ Department of Electronics \& \\ Telecommunication, \\ Sinhgad College of \\ Engineering,Pune,India
}

\author{
D M Bhalerao \\ Department of Electronics \& \\ Telecommunication, \\ Sinhgad College of \\ Engineering,Pune,India
}

\begin{abstract}
Vertical Handoff techniques deal with the scenario in which the mobile node can move between different network access technologies. This paper represents vertical handoff between Wi-Fi and Bluetooth wireless network. When mobile node travels from one network to other network or from one place to other place it require battery power. If the battery power of the mobile is utilized more effectively then it will provide better results in variety of aspects. In paper some parameters of moving mobile node is measured with minimum battery use with the help of virtualization technique. To achieve this goal and to select the best network for mobile node when moving from one network to other network it is also important to have a good utilization of space and access point. Dividing the space into some partitions and assigning access point to each partition helps mobile node in vertical handoff procedure. The combination of virtualization, space and access point is used to apply scalability in vertical handoff.
\end{abstract}

\section{Keywords}

Scalability, virtualization, vertical handoff, Wi-Fi, Bluetooth

\section{INTRODUCTION}

The handoff process has been studied among wireless networks using the same access technology. This kind of handoff is defined as the horizontal handoff scheme the mobile device monitors received power levels and periodically send this information to the MSC controller via their respective BS and which then decides whether a handoff should be performed and if possible then which is target BS. The new handover process among networks using different technologies is defined as vertical handover (VHO). Vertical handover or vertical handoff refers to a network node changing the type of connectivity it uses to access a supporting infrastructure, usually to support node mobility. Vertical handovers refer to the automatic failover from one technology to another in order to maintain communication. This is different from a horizontal handover between different wireless access points that use the same technology in that a vertical handover involves changing the data link layer technology used to access the network [1].

A heterogeneous network is a network connecting computers and other devices with different operating systems and/or protocols. For example, local area networks (LANs) that connect Microsoft Windows and Linux based personal computers with Apple Macintosh computers are heterogeneous. The word heterogeneous network is also used in wireless networks using different access technologies. For example, a wireless network which provides a service through a wireless LAN and is able to maintain the service when switching to a cellular network is called a wireless heterogeneous network. Reference to a HetNet often indicates the use of multiple types of access nodes in a wireless network. A Wide Area Network can use microcells, picocells, and/or femtocells in order to offer wireless coverage in an environment with a wide variety of wireless coverage zones, ranging from an open outdoor environment to office buildings, homes, and underground areas. Mobile Experts defines the HetNet as a network with complex interoperation between microcell, small cell, and in some cases Wi-Fi network elements used together to provide a mosaic of coverage, with handoff capability between network elements[2].

Due to technology changes and high availability of broadband services at high data rates and great improvement in hardware technology components and the usage of internet as a communication media over various entities it is essential to design the actual runtime environment which supports different network changes. Wireless technologies represent a readily emerging area of growth and importance for the communication. In wireless communication Bluetooth, UWB (Ultra Wide Band), ZigBee etc. are used for the shorter communication range. While Wi-MA, UMTS etc. are used for the longer communication[3].

As commonly understood, network selection is to select the best network for a single-homed mobile terminal (MT) or an application of a multi-homed MT in heterogeneous wireless network, which include universal mobile telecommunications system(UMTS), world-wide interoperability for microwave access (Wi-MAX), wireless local area network (WLAN), Bluetooth, etc. To combine multiple criteria together, adjustment (e.g. normalization) of these criteria is required. Meanwhile, certain weighting method (e.g. analytic hierarchy process (AHP)) is required to decide their weights. The selection consider various static and dynamic network-side criteria e.g. bandwidth, jitter, monetary cost, security level, power consumption, traffic load etc. The ranking is done for network on the bases of criteria which is known as ranking criteria [4]. The entire aim is to design the multi criteria decision making (MCDM) algorithm is need to be implement. A key requirement of distributed system is that system developed independently from one another must be able to interoperate with one another. Middleware and network protocol provide common interoperability standards to address this particular channel. In the following figure 1 the framework offers the virtual overlay network which hides the complicated physical network configurations and heterogeneity of the service protocol. In the frame work heterogeneous devices can freely interwork together as if they 
were of the same network type devices. In the framework an Inter-working Gateway (IWG) is defined for the transparent connectivity between heterogeneous networks. Here to select the network side criteria which involves operator policies, terminal prosperities ,customer preferences, application QoS and vertical handover(VHO), horizontal handover (HHO) these all prosperities are known as name factors which depend upon cell radius, coverage percentage, terminal velocity[5].

A key requirement of distributed systems is that systems developed independently from one another must be able to interoperate with one another. However even in this case, developing such interoperability solutions remains laborious and challenging,requiring not only knowledge of the protocols and low-level network programming involved, but also an understanding of the mapping of protocols onto one another or a common intermediary [6]. Handover management issues include mobility scenarios, metrics, decision algorithms and procedures. Mobility scenarios can be classified into horizontal (between different cells of the same network) and vertical (between different types of networks). In homogeneous networks horizontal handovers are typically required when the serving access router becomes unavailable due to MT's movement. In heterogeneous networks, the need for vertical handovers can be initiated for convenience rather than connectivity reasons (e.g., according to user choice for a particular service). Two of the major challenges in vertical handover management are seamlessness and automation aspects in network switching. These particular requirements can refer to the Always Best Connected concept, of being connected in the best possible way in an environment of multiple access technologies, according to policies (expressed by rules based on parameters such as network conditions or user preferences)[7].

\subsection{Classification of Handoff}

In Heterogeneous wireless environment the handoffs are classified into two main streams, Horizontal Handoff (HHO) and Vertical Handoff (VHO). Figure 1 illustrates horizontal and vertical handoff.

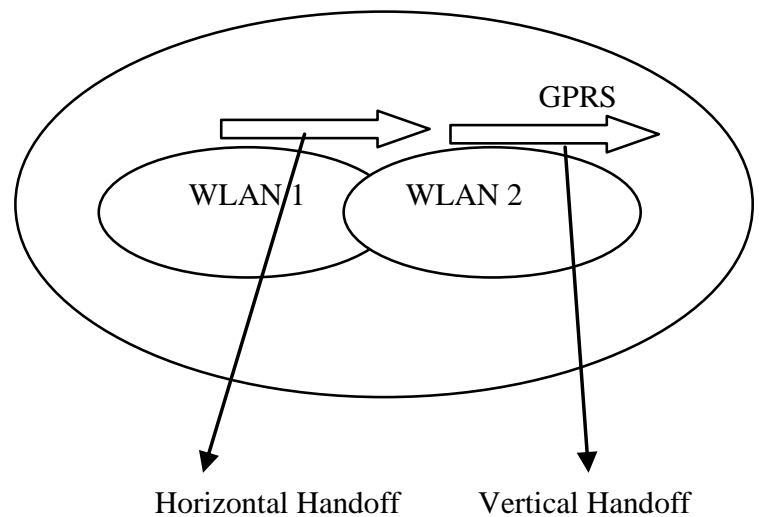

(WLAN 1 to WLAN 2 or vice versa) (WLAN to GPRS)

Figure 1: Horizontal versus vertical handoff

\subsection{Horizontal Handoff}

Horizontal handoff or intra-system handoff is a handoff that occurs between the APs or BSs of the same network technology. A horizontal handoff occurs between the homogeneous cells of a wireless access system. For example, the changeover of signal transmission of an MT from an IEEE 802.11g AP to a geographically neighboring IEEE $802.11 \mathrm{~g}$
AP this process is known as horizontal handoff. The network automatically exchanges the coverage responsibility from one point of attachment to another every time an MT crosses from one cell into a neighboring cell supporting the same network technology. Horizontal handoffs are mandatory since the MT cannot continue its communication without performing it.

\subsection{Vertical Handoff}

The vertical handoff is classified in two categories: upward and downward. The upward vertical handoff is a handoff to a wireless overlay with a larger cell size and lower bandwidth. A downward vertical handoff is a handoff to a wireless overlay with a smaller cell size and larger bandwidth. Thus, a mobile device performing an upward vertical handoff disconnects from a network providing smaller coverage area and higher access speed (for example, WLAN) to a new one providing broader coverage but lower access speed (for example, WWAN), while a mobile device performing a downward vertical handoff disconnects from a network providing broader coverage area and lower access speed to a new one providing limited coverage but higher access speed[8].The main distinguish between Vertical Handoff and Horizontal handoff $(\mathrm{HHO})$ is as shown in table 1.

Table 1: Compression of VHO and HHO

\begin{tabular}{|c|c|c|}
\hline Parameters & VHO & HHO \\
\hline Access Technology & Change & Not changed \\
\hline QoS Parameters & May be changed & Not changed \\
\hline IP Address & Changed & Changed \\
\hline Network Interface & May be changed & Not changed \\
\hline
\end{tabular}

The second classification in vertical handoff mechanism is imperative and alternative. An imperative VHO occurs due to low signal from the base station (BS) or access point (AP). It can be considered as an HHO. The execution of an imperative VHO has to be fast in order to keep on going connections. On the other hand, a VHO initiated to provide the user with better performance is considered to an alternative VHO. This VHO can occur when a user connected to cellular network goes inside the coverage of a WLAN, even if the signal of the connection to the cellular network does not lose any signal strength, the user may consider the connection to WLAN a better option[10].

\section{LITERATURE SURVEY}

\subsection{Vertical Handoff Procedure Overview}

The new generation wireless networks adopt a heterogeneous broadband technology model which aims to provide guarantee seamless connectivity to mobile users, anytime and anywhere. Different network characteristics are expected for different multimedia applications, each of them requiring a specific QoS level. Ubiquitous access through a single network technology could not always guarantee seamless connectivity, due to geographical coverage limitations, so that the cooperation of different access networks represents an important feature for heterogeneous environments. A general definition of handover assumes it as the process by which a mobile terminal keeps its connection active when migrating from the coverage of one network Access Point (AP) to another. Basically, different types of handovers can occur in wireless overlay networks. Network switching can be 
performed not only to maintain user connectivity but also to keep high QoS. There are some decision handover parameters based on QoS, available resources, and channel quality or preference consumer. In GSM, handover decision is based on the perception of channel quality, reflected by the received signal strength and the availability of resources in neighbor cells. The Base Station (BS) usually measures the quality of the radio link channels used by Mobile Nodes (MNs) in its service area. Measures are periodically updated so that degradations in signal strength going below a prescribed threshold can be detected and handover toward another radio channel or cell can be initiated.

Horizontal handover (HHO) occurs between the APs of the same network technology, while vertical handover (VHO) occurs between APs belonging to different networks techonology. Several kind of VHO can be envisaged, as described as follows. A main issue is to decide if or when to start the handover, and who performs it. Handover policies are based on different metrics for handover decision. Traditional solutions simply consider RSSI (Received Signal Strength Indication) and channel availability. More sophisticated handover policies also consider:(i) Quality-of-Service, as different types of services require various combinations of reliability, latency, and data rate; (ii) costs, i.e. different networks may employ different billing strategies; (iii) network conditions like traffic, available bandwidth, network latency, and congestion; (iv) system performance, such as channel propagation characteristics, path loss, inter-channel interference, Signal-to-Noise ratio and Bit Error Rate; (v) mobile terminal conditions like battery power and dynamic factors such as speed, moving pattern, moving histories, and location information. In the latter case, if the battery level of a $\mathrm{MN}$ is low, the handover commutes toward a network that guarantees lower power consumption. In the case when the user requires a guaranteed QoS level for her applications, handover switches to a network meeting such requirements [7].

\subsection{Vertical Handoff Decisions in Heterogeneous Network}

The vertical handoff process can be divided into three main steps namely system discovery, handoff decision, and handoff execution. The function of each step is described below

\subsubsection{System discovery}

During this step, the mobile terminals equipped with multiple interfaces have to determine which networks can be used and the services available in each network.

\subsubsection{Handoff decision}

In this step, the mobile node determines which network it should connect to. The decision may depend on various parameters or handoff metrics Algorithm.

\subsubsection{Interoperability}

In this step, traditional handoff protocols are developed for homogeneous systems that rely on a common signaling protocol, routing techniques and mobility management standard. In heterogeneous environments, mobile nodes and the network routers must be able to interoperate with different networks, and with the corresponding protocols and standards. Traditional handoff protocols are developed for homogeneous systems that rely on a common signaling protocol, routing techniques and mobility management standard. In heterogeneous environments, mobile nodes and the network routers must be able to interoperate with different networks, and with the corresponding protocols and standards [11].

\subsection{Network Virtualization}

Network virtualization is a method of creating independent network topologies as an additional layer on top of the current exiting network architecture. For example, the public Internet is a popular network architecture that forms a baseline infrastructure for a wide array of network virtualization techniques. The Internet provides a high-speed, global network due to its large scale adoption and standardization. The architecture of the Internet provides a natural layering approach that allows protocols and applications to function independently of the layer below. Network virtualization works within this layering approach to provide top level applications the ability to work with the network independent of the actual underlying physical topology. This concept is commonly referred to as cloud computing. Many techniques for providing network virtualization exist. Virtual Private Networking (VPN) is one popular network virtualization technique. The primary purpose of VPNs is to allow the establishment of secure connections between trusted peers on a network. Generally these connections form in such a way as to allow high level network applications to behave as though the other peers in the VPN have connected to the same physical network. In actuality, these peers may be separated by thousands of miles across a complex mesh of networking equipment and interconnection technologies. VPNs usually provide some type of encryption support in order to establish secure tunnels over insecure mediums such as the public Internet [15].

\section{PROPOSED METHOD}

In heterogeneous wireless environment variety of nodes are present. Constraint nodes are one of them which provides internet of things. These nodes have very less energy. If constraint node sends signals continuously; then they became dead after some time. To overcome this limitation of constraint node, virtualization technique is used. Virtualization technique will provide minimum use of battery power for constraint node. Virtualization gives highest priority for the constraint nodes for connection establishment with access point. Whenever constraint node sending a signal for switching onto other network, for example Wi-Fi to Bluetooth. It will get an access by establishing a connection. This paper represents the mechanism by combining virtualization with space and access point. The assumed space (i.e. area) is logically divided into four parts. These partitions can be achieved by assuming a square inside the circular area with the help of figure 2 . The borders of square can be used for handoff procedure. After dividing the assumed area into four parts they require the access points to provide handoff when node is traveling in the various regions. Access point channels are logically allocated to each partition. This entire concept helps to improve scalability in vertical handoff. Each access point is served with a channel. The major job of these access points is to provide highest priority for the signals which are coming from constraint node.

If constraint node comes into respective region of access point and sends signal, it will get priority automatically and vertical handoff takes place. This mechanism provides minimum use of battery which is used by constraint node during movement form one network to other network 


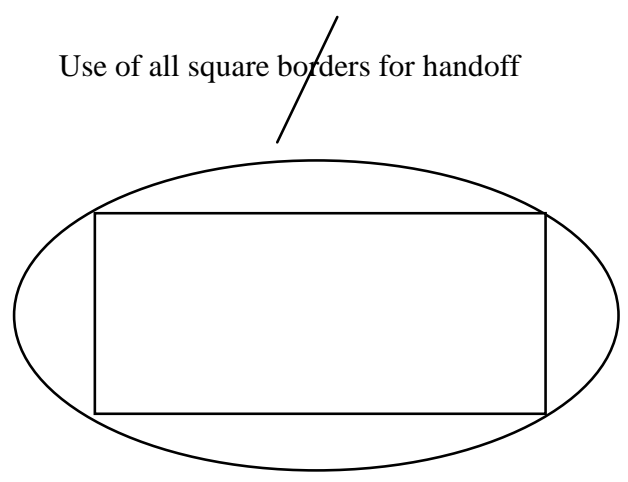

Figure 2: Assumed areas for vertical handoff

As Wi-Fi and Bluetooth network do not belong to the same network technology and therefore a vertical handoff mechanism is required.

In this paper the parameter for measurement are taken against speed of mobile node these are, speed versus packet delivery ratio, speed versus call drop in Bluetooth, speed versus call drop in Wi-Fi, Speed versus power consumed, speed versus

\section{IMPLEMENTATION}

The entire implementation is simulated in NS-2(Network Simulator)

\subsection{Steps for the writing scripts in tcl programming}

Step 1: Creating an instance of the simulator

set ns_[new simulator] //object created

Step 2: Setup trace support by opening file "out.tr" an call the procedure trace-all

set $\mathrm{f}$ [open out.tr w] //log event with record

\$ns trace-all \$f //this file will store all event

Setp3: Setup nf for network animator

set $\mathrm{nf}$ [open out.nam $\mathrm{w}$ ]//this file is assigned to $\mathrm{nf}$ which store relevant data of network animator

Step 4: The topography is broken up into grids and the default value of grid resolution is 1 . A different value can be passed. set topo [new Topography]

\$topo load_flatgrid \$val(x) \$val(y)

set val(chan)

[new Channel/WirelessChannel]

The above four mentioned steps are important to create the instance of the scenario and environment creation.

\subsection{AODV}

The AODV protocol is designed for mobile nodes in ad-hoc networking, where there often are changes in topology. The AODV protocol is based on on-demand route discovery. Because of that every node has different and limited local knowledge of the network. The fact that a node seeks information about the network, only when needed, is causing low overhead since a node does not have to maintaining unnecessary route information. To handle router information AODV uses three different kinds of messages Route request (RREQ), Route Reply (RREP) and Route Error RERR.
AODV is using ring expansion when discovering new routes to limit flooding of the network and thereby reducing overhead.

The protocol is ideal for discovering neighbor nodes. If a node needs a route to a node in the other end of the network1 the protocol will course a reasonable flooding of the network. Expansion ring search is a better strategy than doing a full scale search for the node. Likely some other node in the network has a valid route to the destination, and will send a RREP to source, and thereby reducing overhead. By every RREQ a node sends, a sequence number is increased, this is used by the protocol to guarantee loop-freedom in paths found. New version of the AODV routing protocol has also a feature that only the destination host can reply to the sent request.

\subsection{Creating the object GOD}

Create the object God, "God (General Operations Director) is the object that is used to store global information about the state of the environment, network or nodes. The procedure create-god is defined in \$NS2_HOME/tcl/mobility/com.tcl, which allows only a single global instance of the God object to be created during a simulation. God object is called internally by MAC objects in nodes, so create god in every cases. Table 2 shows the syntax for god configuration.

\section{Table 2: Method of for node configuration}

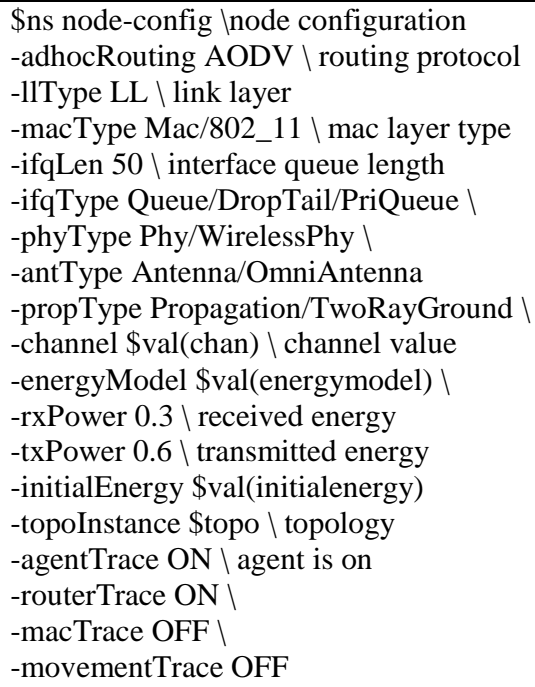

\subsection{Performance parameter to measure scalability in vertical handoff for Wi-Fi and Bluetooth Network}

The performance parameters are measured that can be obtained through NS trace file.

\subsubsection{Speed versus Packet delivery ratio}

It is the ratio of the number of delivered data packet to the destination. The greater value of packet delivery ratio means the better performance of the protocol. As mobile node is moving it is necessary to maintain records of Speed versus Packet delivery for calculation of average packet delivery ratio. Average packet delivery ratio will provide the range of the packet delivery. Due to this range it is easy to calculate the number of packet delivered and number of packet are lost during the movement of node. 


\subsubsection{Speed versus Call drop in Bluetooth}

Call drop in Bluetooth occurs when handover between two sectors is not well defined. If connection speed drops from fast to slow then call drop occurs. To calculate speed versus call drop in Bluetooth the aodv protocol can be used which is present in NS-2. The information of call dropping count is present on access point. measured the call dropping count when node is moving in Bluetooth.

\subsubsection{Speed versus Call drop in Wi-Fi}

Call drop in Bluetooth occurs when handover between two sectors is not well defined. To calculate speed versus call drop in Wi-Fi the aodv protocol is used which is present in NS-2.

\subsubsection{Speed versus Power Consumption}

As node is moving to energy of the respective node is need to be preserved. The measurement of speed versus the power consumed by the node is taken under cosideration. If speed of the node is increasing definitely power consumption by node is reduced.

\subsubsection{Speed versus Handoff Delay}

Handoff delay is the ratio of handoff time by handover count. The handover delay is important when the node is moving form Bluetooth to $\mathrm{Wi}-\mathrm{Fi}$ and vice versa. When the node changes its network this delay is required to provide. Handoff delay is appears random in manner.

\section{RESULTS}

\subsection{Speed versus Packet delivery ratio}

After simulating the proposed method in NS-2 the results are found, which represented in table 3 . The speed is represented in $\mathrm{m} / \mathrm{s}$ and packet delivery is ratio (PDR) is in percentage. Initial speed is $5 \mathrm{~m} / \mathrm{s}$ and the reading is taken up to $25 \mathrm{~m} / \mathrm{s}$. The maximum packet delivery ratio is at speed $10 \mathrm{~m} / \mathrm{s}$ and minimum packet delivery ratio is at $25 \mathrm{~m} / \mathrm{s}$, it is $97.83 \%$ and $83.79 \%$ respectively. This paper shows that the packet delivery ratio is increased if the battery power of the node is utilized effectively with the help of virtualization.

Table 3: Speed versus packet delivery ratio

\begin{tabular}{|c|c|c|}
\hline Sr. No. & Speed(m/s) & Packet Delivery Ratio (\%) \\
\hline 1. & 5 & 88.69 \\
\hline 2. & 10 & 97.83 \\
\hline 3. & 15 & 93.34 \\
\hline 4. & 20 & 87.70 \\
\hline 5. & 25 & 83.79 \\
\hline
\end{tabular}

\subsection{Speed versus Call Drop in Bluetooth}

The table 4 shows results by moving the mobile node from one position to other position. Speed is represented in terms of $\mathrm{m} / \mathrm{s}$ and the respective call dropping count. Call dropping count is totally depends upon the position of the respective destination of node. This is occurred when handover between two sector are not well defined. Here in this paper the CBR (Constant bit rate) traffic is used for the overall communication. In Bluetooth the call dropping count is necessary to prevent the seamless connectivity from one network to other network or one position to other position. The call dropping count is different at the different speed. The maximum call dropping count is 1251 at speed $5 \mathrm{~m} / \mathrm{s}$. Call dropping count in Bluetooth is become less if speed of the of node is increases.

Table 4: Speed versus Call drop in Bluetooth

\begin{tabular}{|c|c|c|}
\hline $\begin{array}{c}\text { Sr. } \\
\text { No. }\end{array}$ & $\begin{array}{c}\text { Speed } \\
(\mathrm{m} / \mathrm{s})\end{array}$ & $\begin{array}{c}\text { Call drop in Bluetooth (Call dropping } \\
\text { count) }\end{array}$ \\
\hline 1. & 5 & 1251 \\
\hline 2. & 10 & 1234 \\
\hline 3. & 15 & 1051 \\
\hline 4. & 20 & 1134 \\
\hline 5. & 25 & 1027 \\
\hline
\end{tabular}

\subsection{Speed versus Call Drop in Wi-Fi}

Table 5 shows speed versus call drop in Wi-Fi. This table is obtained during Wi-Fi node movement. Speed is represented in terms of $\mathrm{m} / \mathrm{s}$ and the respective call dropping count is measured. As the node is moving from one position to other position it important to measure the call dropping ratio. Control information can be known by the control over head which are required in this mechanism. It will provide the data to the respective network call dropping will gives the number of call drop occurs during the node movement. Table provides the exact numeric values which we can easily understand. The maximum call dropping count is 591 at speed $5 \mathrm{~m} / \mathrm{s}$. Call dropping count is found less in Wi-Fi if it is compared with Bluetooth call drop.

Table 5: Speed versus call drop in Wi-Fi

\begin{tabular}{|c|c|c|}
\hline $\begin{array}{c}\text { Sr. } \\
\text { No. }\end{array}$ & $\begin{array}{c}\text { Speed } \\
(\mathrm{m} / \mathrm{s})\end{array}$ & $\begin{array}{c}\text { Call drop in Wi-Fi (Call dropping } \\
\text { count) }\end{array}$ \\
\hline 1. & 5 & 591 \\
\hline 2. & 10 & 558 \\
\hline 3. & 15 & 558 \\
\hline 4. & 20 & 450 \\
\hline 5. & 25 & \\
\hline
\end{tabular}

\subsection{Speed versus Power Consumption}

Table 6 shows the values of speed versus power consumed. These reading are required during node movement. Speed is represented in $\mathrm{m} / \mathrm{s}$ and power consumed is in watt $(\mathrm{W})$. As per the table it is clear that when speed of the mobile node is increasing and power is reduced. At speed $5 \mathrm{~m} / \mathrm{s}$ and $25 \mathrm{~m} / \mathrm{s}$ is $6.13 \mathrm{~W}$ and $5.50 \mathrm{~W}$ respectively. As per the values in the table 6 it clear that if speed of the mobile node is increased then the respective power required for the mobile node is going to be decreased. In short the power consumption is achieved during this scenario. If the less power is utilized during the node movement definitely the life of the node will increased. Due to increase in life of node the entire communication system 
can work efficiently. This entire mechanism automatically improves the performance of the system.

Table 6: Speed versus power consumed

\begin{tabular}{|c|c|c|}
\hline Sr. No. & Speed (m/s) & Power Consumption (Watt) \\
\hline 1. & 5 & 6.13 \\
\hline 2. & 10 & 6.07 \\
\hline 3. & 15 & 5.91 \\
\hline 4. & 20 & 5.61 \\
\hline 5. & 25 & 5.50 \\
\hline
\end{tabular}

\subsection{Speed versus Handoff Delay}

In this method the call dropping count takes an important role that has been already calculated during the node movement in both network i.e. in Bluetooth and Wi-Fi. Handoff mechanism requires when the node is moved from one network to other network. The control over head is again more important. Table 7 represents the values for speed versus handoff delay. Here the speed of the mobile node is represented in $\mathrm{m} / \mathrm{s}$ and the handoff delay is second.

Handoff delay plays a vital role in vertical handoff mechanism for Wi-Fi and Bluetooth network. This delay will provide the related information of the node how much time it will take to reconnect when it moves form one network to the other network. Handoff delay depends upon handoff time and handover count. If handoff time is decreased and handover count is increased handoff delay became less. Lesser handoff delay provides a faster connectivity to the respective nodes.

Table 7: Speed versus handoff delay

\begin{tabular}{|c|c|c|}
\hline Sr. No. & Speed (m/s) & Handoff Delay (second) \\
\hline 1. & 5 & 0.240 \\
\hline 2. & 10 & 0.310 \\
\hline 3. & 15 & 0.242 \\
\hline 4. & 20 & 0.310 \\
\hline 5. & 25 & 0.229 \\
\hline
\end{tabular}

\section{CONCLUSION}

This paper shows scalability in vertical handoff for Wi-Fi and Bluetooth wireless network is simulated against speed of mobile nodes. Virtualization technique helps constraint nodes to use minimum battery power. Power consumption is achieved against the speed of the mobile nodes. After power consumption the packet delivery ratio is improved of mobile nodes. Call dropping count is reduced in both Wi-Fi and Bluetooth network technology against speed of mobile nodes. Handoff delay for mobile nodes is found fluctuating in nature.

\section{REFERENCES}

[1] Anubhuti Khare, Manish Saxena \& Bhagawati Patil," Performance Analysis for Optimization of Vertical Hand-Off in Hetrogeneous Wireless," International Journal on Emerging Technologies 2(1), pp. 140-143, June 2011.

[2] http://www.wikipedia.com/

[3] Lusheng Wang \& David Binet,"Mobility-based Network Selection Scheme in Heterogeneous Wireless Networks," IEEE 2009 vol no 68, pp 1482-1488

[4] Lusheng Wang \& David Binet, " MADM-based Network Selection in Heterogeneous Wireless Networks: A Simulation Study," IEEE 2009 Wireless VITAE'09

[5]Hojin Park, Jun-Hee Parkh \& Nam Kim, "A Framework for Interoperability of Heterogeneous Devices in Ubiquitous Home," IEEE Second International Conference on Advances in Future Internet pp. 50-55 2010.

[6] Y'erom-David Bromberg LaBRI, Paul Grace \& Laurent R'eveill'ere LaBRI," Starlink: runtime interoperability between heterogeneous middleware protocols," IEEE Trans 31st International Conference on Distributed Computing System pp.446-455 2010.

[7] Meriem Kassar, Brigitte Kervella \& Guy Pujolle,"An overview of vertical handover decision strategies in heterogeneous wireless networks,"Science Direct Computer Communications 31 (2008) 2607-2620 January 2008

[8] Akhila S, Jayanthi K Murthy, Arathi R Shankar \& Suthikshn Kumar,"An Overview on Decision Techniques for Vertical Handoffs across Wireless Heterogeneous Networks,", International Journal of Scientific \& Engineering Research, Volume 3, Issue 1, pp.16,January-2012

[9] Bhavneet Sidhu, Hardeep Singh, and Amit Chhabra "Emerging Wireless Standards - WiFi, ZigBee and WiMAX,". World Academy of Science, Engineering and Technology 252007.

[10] Eric A. Brewer, Randy H. Katz, Network Architecture for Heterogeneous Mobile Computing,"

[11] K.Savitha \& C.Chandrasekar, "Trusted Network Selection using SAW and TOPSIS Algorithms for Heterogeneous Wireless Networks" International Journal of Computer Applications (0975 - 8887), Volume 26-No.8, pp. 22-29, July 2011.

[12] Suman Kumar Sikdar, Uttam Kumar Kundu \& Debabrata Sarddar "Minimization of Call Blocking Probability using Mobile Node velocity," International Journal of Advanced Computer Science and Applications, Vol. 3, No. 11 pp. 172-179, 2012.

[13] Ling-Jyh Chen, Tony Sun \& Benny Chen "A Smart Decision Model for Vertical Handoff."

[14] J.Banos, C.Cárdenas \& C.Perez "Testing of wireless heterogeneous networks," IEEE MELECON 2006.

[15] Kyle E. Stewart, Todd R. Andel, and Jeffrey W. Humphries "Measuring the Performance of Network Virtualization Tool N2N in the Design of a Cyber Warfare Training and Education Platform" 